\title{
Dual representation of dipole photons
}

\author{
Alexander S Shumovsky \\ Physics Department, Bilkent University, Bilkent, 06533 Ankara, Turkey
}

Received 20 April 1999

\begin{abstract}
The quantum polarization and phase properties of an electric dipole radiation are examined. It is shown that, unlike the classical picture, the quantum description of polarization needs nine independent Stokes operators, forming a representation of SU(3) sub-algebra in the Weyl-Heisenberg algebra of photons. A corresponding Cartan algebra determines the cosine and sine of the radiation phase operators. A new representation of dipole photons is proposed. The generatorsoftheCartanalgebraarediagonalinthisrepresentationsothatthecorrespondingnumber states describe the number of photons with given radiation phase. The eigenstates of the radiation phase are determined. They have a discrete spectrum and natural behaviour in the classical limit. The relation between the radiation phase, operational phases and the Pegg-Barnett approach is discussed.
\end{abstract}

\section{Introduction}

It is well known that quantum electrodynamics describes a pure multipole radiation generated by an atomic or molecular transition in terms of 'spherical' photons with given angular momentum and parity [1]. The angular momentum JE of a quantum mechanical system is usually specified by a representation of the $\mathrm{SU}(2)$. If the corresponding enveloping algebra contains a uniquely defined scalar (the Casimir operator), the polar decomposition of JEcan be obtained [2]. This polar decomposition determines a dual representation of $\mathrm{SU}(2)$ expressed in terms of so-called phase states [2], describing the quantum phase of the angular momentum.

Although the angular momentum ME of a multipole radiation is well defined in terms of the spherical photons [1], the polar decomposition of ME is impossible. The reason is that ME is represented by generators of an $\mathrm{SU}(2)$ sub-algebra which has no isotype representation [3] in the Weyl-Heisenberg algebra of photon operators. This means that the Casimir operator cannot be uniquely determined in the whole Hilbert space HR of photon states. Hence, the quantum phase of the angular momentum of a multipole radiation cannot be determined by the method of [2] valid for the quantum mechanical systems.

An approach focused on overcoming this difficulty has been proposed recently [4]. The main idea, which seems to be a very natural one, is to consider the radiation of a given quantum source rather than a free electromagnetic field. Even in the classical picture, the multipole radiation can be determined completely only if the source functions, describing a localized source at the origin, are known (e.g., see [5]). Within the quantum picture, we can take into consideration the 'source dependence' of radiation in the following way. Since the total angular momentum JE $+\mathrm{ME}$ is conserved in the process of generation, we can first construct the polar decomposition of JE in the $(2 j+1)$-dimensional atomic Hilbert space $\mathrm{HA}_{\mathrm{A}}$, following the method by Vourdas [2]. Then, for the 'phase-dependent' dual representation of theatomicSU(2)algebra, wehavetodeterminetheradiationcounterpart, whichconsistsofthe

operators complementary to the atomic operators with respect to the integrals of motion $[4,6]$. In particular, the cosine and sine of the so-called radiation phase have been determined in this way for the electric dipole radiation by a two-level atom $[4,6]$. The radiation phase is, by construction, the quantum phase of angular momentum of radiation. 
Many attempts have been made to define the quantum phase of light via the angular momentum (e.g., see [7]). The new element of our approach [4] is that we determine the quantum phase of radiation via the quantum phase of angular momentum of its source. We also note that one of the most popular and important methods in the field of quantum phase, proposed by Pegg and Barnett [8] (for a recent review, see [9]), is based on a contraction of the infinite-dimensional Hilbert space of photon states Hr. Within this method, the quantum phase variable is determined first in an s-dimensional ( $\mathrm{s}$ is a finite integer) sub-space of HR, and the formal limit $\mathrm{s} \rightarrow \infty$ is taken only after the averages have been calculated. In contrast, we consider an extended space of states $^{H_{A} \otimes H R}$ in which the quantum phase of radiation is determined by mapping of corresponding operators from $\mathrm{HA}$ into $\mathrm{HR}$, using the conservation law. By definition, the radiation phase is expressed in terms of what can be generated by a given quantum source. In some sense, our approach is complementary to that proposed by Mandel et al [10], in which the quantum phase is determined in terms of what can be measured.

Inrecentpublications[11] wehaveshownthattheradiationphaseasdefinedin[4]hasclose connectionwiththegeneralizedStokesoperatorsintroducedin[12] todescribethepolarization propertiesoftheelectricdipoleradiationinthequantumdomain. Thisisnotsurprising,because the radiation phase describes the quantum phase of angular momentum which, generally speaking, consists of the spin and 'orbital' contributions, while the polarization is defined to be a given spin state of photons (e.g., see [13]). We note that some attempts to determine the quantum phase (or phase difference) in terms of polarization are known [14]. The polarization has been described in [14] by conventional Stokes operators of a monochromatic plane wave (free electromagnetic field) and the quantum phase variable has been defined in the spirit of the Pegg-Barnettapproach[8,9]. Thispictureofpolarizationdealswiththetransversalanisotropy oftheelectricfield. Inthecaseofmultipoleradiation, inadditiontothetransversalcomponents thepolarizationalwayshastheradialcomponent, atleastinthenearandintermediatezones[5], and the spatial anisotropy should be considered rather than the transversal one. In spite of the factthattheradialcomponentusuallyhaslowintensityatfardistances, itmaystronglyinfluence the quantum fluctuations of the polarization of the two other components [12]. Therefore it cannot be neglected, even in the vacuum state.

In the present paper we continue the discussion of the radiation phase [4]. We complete the quantum description of the polarization of electric dipole radiation. We examine the spectrum of the cosine and sine of radiation phase operators and show that the eigenstates of these operators are certain new Fock number states, forming a dual representation with respect to the conventional number states of dipole photons. In section 2 , based on our previous results $[4,6,11,12]$, we briefly discuss the 'transmission' of quantum phase information from the atomic angular momentum to photons. In section 3 we consider the quantum polarization properties of the electric dipole radiation in the quantum domain. It is shown that the total set of Stokes operators is determined in this case by the nine generators of the SU(3) subalgebra in the Weyl-Heisenberg algebra of dipole photons. The Cartan algebra of this SU(3) determines the cosine and sine of the radiation phase operators. In section 4 we introduce the dual representation of the dipole photons via some canonical transformation and then define the radiation phase states as the number states of dual photons. In section 5 we discuss the cosine and sine of the radiation phase operators. We conclude in section 6 with a discussion of our results. 


\section{Transmission of quantum phase information between atom and photons}

Following [6], consider a two-level atom with the electric dipole transition between the levels with angular momenta $\mathrm{j}=1$ and $\mathrm{j}^{0}=0$, situated at the centre of an ideal spherical cavity. The excited atomic state $\mathrm{j}=1 ; \mathrm{m}=0, \pm 1 \mathrm{i}$ is triple degenerated in this case. In the process of radiation, a photon takes away the angular momentum $\mathrm{j}=1$ and projection $\mathrm{m}=0, \pm 1$ of the corresponding sub-level of the excited atomic state. If we introduce the atomic operators Rmmo $=\|$ mihmo $\|$, where

$$
\| m\rangle \equiv|j=1 ; m\rangle
$$

the angular momentum $\mathrm{J}$ of the excited state can be represented by the following generators of the $\mathrm{SU}(2)$ algebra [6]:

$$
\begin{aligned}
& J_{z}=R_{++}-R_{--} \\
& J_{+}=\sqrt{2}\left(R_{+0}+R_{0-}\right) \\
& J_{-}=\sqrt{2}\left(R_{0+}+R_{-0}\right)
\end{aligned}
$$

so that $\left[\mathrm{J}_{+}, \mathrm{J}_{-}\right]=2 \mathrm{~J}_{\mathrm{z}},\left[\mathrm{J}_{\mathrm{z}} \mathrm{J}_{ \pm}\right]= \pm \mathrm{J}_{ \pm}$, and

$$
J E^{2}=j(j+1) X R_{m m}=2 \times \mathbf{1}_{A}
$$

$$
\mathrm{m}=-1
$$

where $\mathbf{1}_{\mathrm{A}}$ is the unit operator in the three-dimensional space of states (1). Then, by performing ananalysissimilartothatproposedbyVourdas[2], onecanintroducethefollowingexponential of the quantum phase operator:

$$
\mathrm{EA}=\mathrm{R}_{+0}+\mathrm{R}_{0-}+\mathrm{R}_{-+} \quad \mathrm{EAEA}^{+}=\mathbf{1}_{\mathrm{A}} \quad \mathrm{EA}_{3}=\mathrm{EA}
$$

the eigenstates of which

$$
\left.\left.\mathrm{EA}\left\|\varphi \mathrm{mi}=\mathrm{ei} \varphi_{\mathrm{m}}\right\| \varphi \mathrm{mi} \quad \phi_{m}=\frac{2 m \pi}{3} \quad \mathrm{~m}=0, \pm 1 \quad \| \phi_{m}\right\rangle=\frac{1}{\sqrt{3}} \sum_{m^{\prime}=-1}^{1} \mathrm{e}^{-\mathrm{i} m^{\prime} \phi_{m}} \| m^{\prime}\right\rangle
$$

determine the basis in HA dual to (1). States (5) are usually called the phase states [2]. The phase variable determined via the exponential of the phase operator (4) describes the azimuth (longitude) of the angular momentum of the excited atomic state.

Any photon generated by the electric dipole transition under consideration has given angular momentum $\mathrm{j}=1$, projection $\mathrm{m}$ and parity $\mathrm{P}=-1$. It is described by the annihilation and creation operators $\mathrm{a}_{\mathrm{m}}$ and $\mathrm{am}_{\mathrm{m}}{ }^{+}[1]$ determined in the Hilbert space

$$
\begin{gathered}
\mathrm{O}_{(\mathrm{m})}^{1} \\
\mathrm{HR}=\mathrm{HR} . \\
\mathrm{m}=-1
\end{gathered}
$$

Here the infinite-dimensional sub-space $\mathrm{HR}^{(\mathrm{m})}$ is spanned by the countable set of Fock vectors 
$\mid \mathrm{n}_{\mathrm{m}} \mathrm{i}, \mathrm{n}_{\mathrm{m}}=0,1,2, \ldots$, which obey the orthogonality condition $\left\langle n_{m}^{\prime} \mid n_{m}\right\rangle=\delta_{m m^{\prime}} \delta_{n n^{\prime}}$ and the completeness condition [1]

$$
\bigotimes_{m=-1}^{1} \sum_{n_{m}=0}^{\infty}\left|n_{m}\right\rangle\left\langle n_{m}\right|=\mathbf{1}
$$

where $\mathbf{1}$ is the unit operator in (6). The photon operators form in (6) a representation of the Weyl-Heisenberg algebra with the commutation relation

$$
[\mathrm{am}, \mathrm{am}+0]=\mathbf{1} \delta \mathrm{mm} 0 .
$$

The angular momentum ME of the electric dipole radiation is represented by the operators $($ see $[1])$

$$
\begin{aligned}
& M_{z}=\sum_{m=-1}^{1} m a_{m}^{+} a_{m} \\
& M_{+}=\sqrt{2}\left(a_{+}^{+} a_{0}+a_{0}^{+} a_{-}\right) \\
& M_{-}=\sqrt{2}\left(a_{0}^{+} a_{+}+a_{-}^{+} a_{0}\right)
\end{aligned}
$$

forming a representation of the SU(2) sub-algebra in the Weyl-Heisenberg algebra (8). Due to the conservation of angular momentum in the process of radiation, the operators $J_{p}+M_{p}(p$ $=\mathrm{z}, \pm$ ) are the integrals of motion with respect to the modified Jaynes-Cummings Hamiltonian [6]

$$
H=\sum_{m=-1}^{1}\left[\omega a_{m}^{+} a_{m}+\omega_{0} R_{m m}+g\left(R_{m G} a_{m}+a_{m}^{+} R_{G m}\right)\right.
$$

which describes the system under consideration. Here $\omega$ is the frequency of the cavity photons, $\omega_{0}$ is the atomic transition frequency, $\mathrm{g}$ is the coupling constant, and $\mathrm{RGm} \equiv \mid \mathrm{j} 0=0 ; \mathrm{m} 0=0 \mathrm{ihm} \|$. It is clear that $\mathrm{ME}^{2}$ determined by (9) is not a C-number in (6) (it is not proportional to the unit operator (7)). Hence, the polar decomposition of (9) cannot be determined in the way proposed in [2] for the quantum mechanical systems.

At the same time, one can define the operator $[4,6,11]$

$$
\mathcal{E}=a_{+}^{+} a_{0}+a_{0}^{+} a_{-}+a_{-}^{+} a_{+}
$$

which complements the atomic exponential of the quantum phase operator (5) with respect to the integral of motion with the Hamiltonian $(10)\left[\left(E_{A}+E\right), H\right]=0$.

Unlike (5), it is not unitary, but it is normal:

$$
\left[\mathrm{E}, \mathrm{E}^{+}\right]=0 \text {. }
$$

The physical meaning of operator (11) is discussed in the next sections.

\section{Polarization}

Before we begin to discuss the dual representation of the photon operators, consider the quantum polarization properties of the electric dipole radiation, both classical and quantum. In the case of a classical monochromatic electric dipole radiation, only the induction $\mathrm{BE}$ is 
always orthogonal to the direction of propagation (the radial direction of outgoing spherical waves), while the electric field

$$
\vec{E}=\sum_{m=-1}^{1}\left[\frac{\mathrm{i}}{k} b_{m} \vec{\nabla} \times f(k r) \vec{Y}_{1 m}\right] \equiv \sum_{m=-1}^{1} \vec{E}_{m}
$$

has both radial and transversal components [5]. Here the coefficients $b_{m}$ are determined by the source, $\mathrm{f}(\mathrm{kr})$ is a certain combination of the Hankel functions describing the radial dependence, and YE is the vector spherical harmonics describing the angular dependence of radiation. The component EE0 of field (13) describes a linearly polarized radial (longitudinal) field, while $\vec{E}_{ \pm}$describe the circularly polarized components with positive and negative helicities.

Following the standard method [15], one can define the $3 \times 3$ Hermitian matrix of polarization whose elements are [12]

$$
\mathcal{P}_{m m^{\prime}}=E_{m}^{*} E_{m^{\prime}} \text {. }
$$

Clearly, this is a generalization of the conventional polarization matrix determined in the circular polarization basis [15], which can be obtained from (14) at $\mathrm{E}_{0}=0$. It is a straightforward matter to arrive at the conclusion that the nine elements of the polarization matrix (14) can be completely determined by only five real parameters: precisely, by the three intensities $\mathrm{I}_{\mathrm{m}}=$ Pmm and any two of the five classical phase differences

$$
1 \mathrm{~mm} 0 \equiv \operatorname{argEm}-\operatorname{argEmo}
$$

such that

$$
1+0+10^{-}+1-+=0
$$

Then, the generalized Stokes parameters of the electric dipole radiation can be chosen as follows [12]:

$$
\begin{aligned}
s_{0} & =\sum_{m} E_{m}^{*} E_{m} \\
s_{1} & =2 \operatorname{Re}\left(E_{+}^{*} E_{0}+E_{0}^{*} E_{-}+E_{-}^{*} E_{+}\right) \\
s_{2} & =2 \operatorname{Im}\left(E_{+}^{*} E_{0}+E_{0}^{*} E_{-}+E_{-}^{*} E_{+}\right) \\
s_{3} & =E_{+}^{*} E_{+}-E_{-}^{*} E_{-} \\
s_{4} & =E_{+}^{*} E_{+}+E_{-}^{*} E_{-}-2 E_{0}^{*} E_{0} .
\end{aligned}
$$

They have very simple physical meaning. The parameter $\mathrm{s}_{0}$ measures the total intensity, $\mathrm{s}_{1}$ and $\mathrm{s}_{2}$ give the phase information, $\mathrm{s}_{3}$ gives the preponderance of the positive helicity over the negative one, and the parameter $\mathrm{s}_{4}$ gives the preponderance of the linear (radial) polarization over the two circular (transversal) ones.

Using the canonical quantization of the multipole field [1], we can obtain from (15) the following generalized Stokes operators [12]:

$$
\begin{gathered}
S_{0}=X a_{m}+a_{m} \\
m \\
S_{1}=\left(E+E^{+}\right)
\end{gathered}
$$




$$
\begin{aligned}
& \mathrm{S}_{2}=-\mathrm{i}\left(\mathrm{E}-\mathrm{E}_{+}\right) \\
& \mathrm{S}_{3}=\mathrm{a}_{+} \mathrm{a}_{+}-\mathrm{a}_{+-} \mathrm{a}_{-} \quad+ \\
& \mathrm{S}_{4}=\mathrm{a}+\mathrm{a}++\mathrm{a}-\mathrm{a}_{-}-2 \mathrm{a} 0 \mathrm{a} 0 .
\end{aligned}
$$

Here the operator $\mathrm{E}$ is determined by equation (11) and in view of (12) we get

$$
\left[\mathrm{S}_{1}, \mathrm{~S}_{2}\right]=\left[\mathrm{S}_{1}, \mathrm{~S}_{0}\right]=\left[\mathrm{S}_{2}, \mathrm{~S}_{0}\right]=0(17) \text { i.e., that corresponding physical quantities }
$$
can be measured simultaneously. At the same time $\left[S_{1,2}, S_{3,4}\right] 6=0$, which implies corresponding uncertainty relations.

WenownotethatthetotalsetofindependentHermitianbilinearformsunderconsideration is represented by the following generators of the SU(3) sub-algebra in the Weyl-Heisenberg algebra (8):

$$
\begin{array}{lcc}
\left(a_{+}^{+} a_{+}-a_{0}^{+} a_{0}\right) & \left(a_{0}^{+} a_{0}-a_{-}^{+} a_{-}\right) & \left(a_{-}^{+} a_{-}-a_{+}^{+} a_{+}\right) \\
\frac{1}{2}\left(a_{+}^{+} a_{0}+a_{0}^{+} a_{+}\right) & \frac{1}{2}\left(a_{0}^{+} a_{-}+a_{-}^{+} a_{0}\right) & \frac{1}{2}\left(a_{-}^{+} a_{+}+a_{+}^{+} a_{-}\right) \\
\frac{1}{2 \mathrm{i}}\left(a_{+}^{+} a_{0}-a_{0}^{+} a_{+}\right) & \frac{1}{2 \mathrm{i}}\left(a_{0}^{+} a_{-}-a_{-}^{+} a_{0}\right) & \frac{1}{2 \mathrm{i}}\left(a_{-}^{+} a_{+}-a_{+}^{+} a_{-}\right) .
\end{array}
$$

One can easily see that the five operators (16) can be expressed in terms of linear combinations of different generators (18). Moreover, the operators $S_{1}$ and $S_{2}$ form the Cartan algebra in the SU(3) algebra (18). But generators (18) contain much more information than (16). At the same time, it is clear that (18) looks like a representation of the polarization matrix (14) in the quantum domain. Unlike the classical case, the elements of the quantum polarization matrix are specified by nine independent operators. Therefore, the number of independent Stokes operators also should be equal to nine. This means that, in addition to operators (16), which represent the quantum counterpart of the Stokes parameters (15), we have to introduce four more Stokes operators. For example, the 'additional' Stokes operators can be chosen as

$$
\begin{gathered}
\mathrm{S}_{5}=\left(\mathrm{a}_{+}^{+} \mathrm{a}^{-}+\mathrm{a}^{+} \mathrm{a}_{++}\right) \\
+ \\
\mathrm{S}_{6}=-\mathrm{i}\left(\mathrm{a}+\mathrm{a}^{-}-\mathrm{a}-\mathrm{a}+\right) \\
+++ \\
\mathrm{S}_{7}=\left(\begin{array}{c}
+ \\
\mathrm{a}++
\end{array}+\mathrm{a}+\mathrm{a} 0\right) \\
\mathrm{S}_{8}=-\mathrm{i}(\mathrm{a} 0+\mathrm{a}++\mathrm{a}++\mathrm{a} 0) .
\end{gathered}
$$

The operators $S_{3}$ in (16) and $S_{5}, S_{6}$ in (19) are seen to form a representation of the $S U(2)$ subalgebra, apart from an inessential factor. They formally coincide with the conventional Stokes operators [14] determined for a monochromatic plane wave in the circular polarization basis. Let us stress the formal character of this coincidence. The operators $a_{m}, a_{m}^{+}$in (16) and (19) describe the spherical photons with given angular momentum $\mathrm{j}=1$ and projection $\mathrm{m}$, while the photon operators in [14] are determined for the states with given linear momentum.

We now note that, in the important case of the vacuum radial field, the Stokes operators $S_{1}$ and $S_{2}$ from (16) and $S_{5}$ and $S_{6}$ from (19) describe the same values of the Stokes parameters. Nevertheless, the operators $S_{1,2}$ and $S_{5,6}$ describe absolutely different physical quantities. To 
illustrate this fact, consider the state $\mid \alpha_{+} ; 0 ; \alpha-i$ of the radiation field, so that the two circularly polarized components are in coherent states each, while the radial component is in the vacuum

state.

$$
\left\langle S_{1}\right\rangle=\left\langle S_{5}\right\rangle=2|\alpha| \cos \Delta_{+-}
$$

$$
\text { Assume for simplicity that } 2 \text {. }\left|\alpha_{+}\right|=\left|\alpha_{-}\right| \equiv|\alpha| \text {. }
$$

Then

$$
\left\langle S_{2}\right\rangle=\left\langle S_{6}\right\rangle=2 \mid \alpha^{2} \sin \Delta_{+}
$$

where $1_{+^{-}} \equiv \arg \alpha_{+}-\arg \alpha_{-}$. Hence, the mean values of the physical quantities given by $S_{1}$ and $\mathrm{S}_{2}$ (respectively, $\mathrm{S}_{5}$ and $\mathrm{S}_{6}$ ) coincide. At the same time, the variances of the corresponding physical quantities are

$$
\begin{aligned}
& V\left(S_{1}\right)=2|\alpha|^{2}\left(2+\cos \Delta_{+-}\right) \\
& V\left(S_{2}\right)=2|\alpha|^{2}\left(2-\cos \Delta_{+-}\right)
\end{aligned}
$$$$
V\left(S_{5}\right)=V\left(S_{6}\right)=2|\alpha|^{2} . \quad \text { (21) Hence, in spite of the equalities (20), the }
$$
quantum fluctuations (21) of the physical quantities described by $S_{1,2}$ in (16) are much stronger than those described by $S_{5,6}$ in (19). Moreover, they are qualitatively different because of the phase-difference dependence of the first two variances in (21). As a matter of fact, the operators $S_{5,6}$, as well as $S_{7,8}$ in (19), give some extra phase information in addition to that described by the operators $S_{1,2}$ in (16). The evident advantage of the operators $S_{1,2}$ is that they can be measured simultaneously.

\section{Dual representation of photon operators}

Turning back to the definition of the exponential of the quantum phase of the atomic angular momentum operator (3), we note that the dual representation in terms of the phase states (4) leads to the diagonal form of $\mathrm{EA}_{\mathrm{A}}$

$$
\left.\mathcal{E}_{A}^{(\phi)}=\sum_{m} \mathrm{e}^{\mathrm{i} \phi_{m}}|| \phi_{m}\right\rangle\left\langle\phi_{m} \| \equiv \sum_{m} \mathrm{e}^{\mathrm{i} \phi_{m}} R_{m m}^{(\phi)} .\right.
$$

The operator E determined by (11) as the quantum counterpart of EA is not diagonal in the representationofsphericalphotons. However,itcanbediagonalizedbythefollowingcanonical transformation:

$$
\begin{aligned}
& a_{m}=\frac{1}{\sqrt{3}} \sum_{\mu=-1}^{1} \mathrm{e}^{-\mathrm{i} \mu \phi_{m}} A_{\mu} \\
& A_{\mu}=\frac{1}{\sqrt{3}} \sum_{m=-1}^{1} \mathrm{e}^{\mathrm{i} m \phi_{\mu}} a_{m}
\end{aligned}
$$

where

$$
\left[\mathrm{A}_{\mu}, \mathrm{A}+\mu 0\right]=\mathbf{1} \delta_{\mu \mu \nu} .
$$

$\varphi_{m}=(2 m \pi) / \sqrt{3}$ is the same 'phase angle' as in (5), and $\mathbf{1}$ is determined by (7). Then, instead of (11) we get

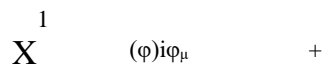




$$
\mathrm{E}=\mathrm{e} \quad \mathrm{A}_{\mu} \mathrm{A}_{\mu} .
$$

Certainly, $A_{\mu}$ and $A_{\mu}^{+}$determine a new representation of the dipole photons. In view of the analogy with the atomic operators, provided by the integrals of motion

$$
[(\mathrm{EA}+\mathrm{E}), \mathrm{H}]=0 \quad\left[\left(\mathrm{EA}^{(\varphi)}+\mathrm{E}^{(\varphi)}\right), \mathrm{H}\right]=0
$$

one can choose to interpret $\mathrm{A}_{\mu}$ and $\mathrm{A}_{\mu}^{+}$as the annihilation and creation operators of the dipole photons with given radiation phase. This radiation phase is the radiation counterpart of the quantum phase of the atomic angular momentum. It is clear that the operators $\mathrm{S}_{0,1,2}$ are also diagonal in the representation (22):

$$
\begin{aligned}
S_{0}^{(\phi)} & =\sum_{\mu} A_{\mu}^{+} A_{\mu} \\
S_{1}^{(\phi)} & =2 \sum_{\mu} A_{\mu}^{+} A_{\mu} \cos \phi_{\mu} \\
S_{2}^{(\phi)} & =2 \sum_{\mu} A_{\mu}^{+} A_{\mu} \sin \phi_{\mu}
\end{aligned}
$$

while all the other operators in (16) and (19) are not.

As can be seen from (22), the operators $A_{\mu}$ obey the same stability condition as $a_{m}$

$$
\left.\forall \mu, \mathrm{m} \quad \mathrm{a}_{\mathrm{m}} \mid \text { vaci }=\mathrm{A}_{\mu} \mid \text { vaci }=0 \quad \mid \text { vac }\right\rangle \equiv \bigotimes_{m}\left|0_{m}\right\rangle .
$$

Hence, $\mathrm{A}^{+}{ }_{\mu}$ can be used to generate the Fock number states

$$
\left|v_{\mu} \mathrm{i}=\left(v_{\mu} !\right)^{-1 / 2}\left(\mathrm{~A}_{\mu}^{+}\right)^{v_{\mu}}\right| \text { vaci }
$$

such that

$$
\mathrm{A}^{+}{ }_{\mu} \mathrm{A}_{\mu}\left|v_{\mu} \mathrm{i}=v_{\mu}\right| v_{\mu} \mathrm{i} \quad v_{\mu}=0,1,2, \ldots
$$

and

$$
\left\langle v_{\mu} \mid v_{\mu^{\prime}}^{\prime}\right\rangle=\delta_{\mu \mu^{\prime}} \delta_{v v^{\prime}} \quad \bigotimes_{\mu=-1}^{1} \sum_{v_{\mu}}\left|v_{\mu}\right\rangle\left\langle v_{\mu}\right|=\mathbf{1}
$$

with the same unit operator as in (7). Hence, the states (25) form a basis in the Hilbert space (6), as well as the states $\mid n_{m} \mathrm{i}$. In analogy to the atomic phase states (4) and, in general, with the quantum phase states introduced in [2], we call (25) the radiation phase states. It follows from (23) and (24) that the radiation phase states (25) are the eigenstates of the operators $\mathrm{E}^{(\varphi)}$, $S_{0}^{(\phi)}$, and $\mathrm{S}_{1(\varphi), 2}$ :

$$
\begin{gathered}
\mathrm{E}^{(\varphi)}\left|v_{\mu \mathrm{i}}=v_{\mu} \mathrm{e} \quad\right| v_{\mu \mathrm{i}} \\
\mathrm{S} 0(\varphi)\left|\nu_{\mu} \mathrm{i}=\nu_{\mu}\right| v_{\mu} \mathrm{i}=\mathrm{S}_{0}
\end{gathered}
$$

$(\varphi)$

$$
\mathrm{S}_{1}\left|v_{\mu} \mathrm{i}=2 v_{\mu} \cos (2 \mu \pi / 3)\right| v_{\mu} \mathrm{i}
$$




$$
\stackrel{(\varphi)}{\mathrm{S}_{2}\left|v_{\mu} \mathrm{i}=2 v_{\mu} \sin (2 \mu \pi / 3)\right| v_{\mu} \mathrm{i} .}
$$

The physical meaning of the radiation phase states can be illustrated with the aid of the Jaynes-Cummings model of section 2. If the two-level atom described by the Hamiltonian (10) is prepared in one of the atomic quantum phase states (4), it generates the dipole photon which can be observed in the state $\mid 1_{\mu}$ i described by (25) at $\mu=\mathrm{m}$. Then, the Stokes operators $S_{1,2 \text { in }}^{(\phi)}(26)$, apart from a factor of two, give the cosine and sine of the quantum phase of the angular momentum.

The above results lead to the conclusion that the radiation phase states (25) are dual with respect to conventional number states $\mid \mathrm{n}_{\mathrm{m}} \mathrm{i}$, like the atomic quantum phase states $\| \varphi_{\mathrm{m}} \mathrm{i}$ (4) and conventional atomic states $\| \mathrm{mi}$ (1). In turn, the operators $A_{\mu}$ and $A_{\mu}^{+}$in (22) form the representation of the Weyl-Heisenberg algebra of the dipole photons dual to the operators $\mathrm{a}_{\mathrm{m}}$ and $a_{m}^{+}$.

Although the canonical transformation (22) has the very simple form of the finite Fourier transformation, the connection between the conventional number states and the radiation phase states (25) is not simple:

$\left|v_{\mu}\right\rangle=\sqrt{\frac{v_{\mu} !}{3^{v_{\mu}}}} \sum_{n_{0}=0}^{v_{\mu}} \sum_{n_{+}=0}^{v_{\mu}-n_{0}} \frac{\exp \left[\mathrm{i}\left(v_{\mu}-n_{0}-2 n_{+}\right) \phi_{\mu}\right]}{\sqrt{n_{0} ! n_{+} !\left(v_{\mu}-n_{0}-n_{+}\right) !}}\left|n_{+} ; n_{0} ; v_{\mu}-n_{+}-n_{0}\right\rangle$.

It is interesting that the 'dual' coherent states

$$
\begin{aligned}
\mid \alpha(\mathrm{a}) \mathrm{i}= & \mathrm{YDm(a)}(\alpha(\mathrm{a})) \mid \text { vaci } \quad \operatorname{Dm}(\mathrm{a})(\alpha(\mathrm{a})) \equiv \exp \left(\alpha_{\mathrm{mam}+}-\text { H.c. }\right) \\
& \mathrm{Y}_{\mathrm{m}} \\
& =\mathrm{D}_{\mu(\mathrm{A}) \mathrm{A})(\alpha(\mathrm{A})) \mid \text { vaci }} \\
& D_{\mu}^{(A)}\left(\alpha^{(A)}\right) \equiv \exp \left(\alpha_{\mu}^{(A)} A_{\mu}^{+}-\text {H.c. }\right)_{\mu}
\end{aligned}
$$

are equivalent to within the following renormalization of the parameters:

$$
\begin{aligned}
& \alpha_{m}^{(a)}=\frac{1}{\sqrt{3}} \sum_{\mu} \mathrm{e}^{-\mathrm{i} \mu \phi_{m}} \alpha_{\mu} \\
& \alpha_{\mu}^{(A)}=\frac{1}{\sqrt{3}} \sum_{m} \mathrm{e}^{\mathrm{i} m \phi_{\mu}} \alpha_{m}^{(a)} .
\end{aligned}
$$

Ifweconsiderasanexamplethesamestate $\mid \alpha_{+} ; 0 ; \alpha$-iofthedipoleradiationasattheendofthe previous section, we will see that it is represented by the dual coherent state $\left|\alpha_{+}^{(A)} ; \alpha_{0}^{(A)} ; \alpha_{-}^{(A)}\right\rangle_{\text {with }}$

$$
\begin{aligned}
& \alpha_{+}^{(A)}=\frac{1}{\sqrt{3}}\left(\alpha_{+} \mathrm{e}^{\mathrm{i} 2 \pi / 3}+\alpha_{-} \mathrm{e}^{-\mathrm{i} 2 \pi / 3}\right) \\
& \alpha_{0}^{(A)}=\frac{1}{\sqrt{3}}\left(\alpha_{+}+\alpha_{-}\right) \\
& \alpha_{-}^{(A)}=\frac{1}{\sqrt{3}}\left(\alpha_{+} \mathrm{e}^{-\mathrm{i} 2 \pi / 3}+\alpha_{-} \mathrm{e}^{\mathrm{i} 2 \pi / 3}\right)
\end{aligned}
$$

in which all the three 'phase' components of the dipole radiation are in the coherent states. 


\section{Phase states and radiation phase}

Taking into account the above results and especially relations (24) and (26), one can choose to interpret the Stokes parameters corresponding to the operators $S_{1,2}$ in (16) or $S_{1,2}^{(\phi)}$ in (24) as the non-normalized cosine and sine of the radiation phase. It should be stressed that this interpretation follows the standard ideology of the classical definition of the cosine and sine of the phase difference between two circularly polarized components $[5,15]$ and the operational definition of the cosine and sine operators of the quantum phase [10]. Consider the state

$$
|\phi\rangle=\bigotimes_{\mu=-1}^{1}\left|v_{\mu}\right\rangle
$$

where $\mid v_{\mu} \mathrm{i}$ are the radiation phase states (25). It is clear that (29) is the eigenstate of the operators (23) and (24). Since the operators $\mathcal{E}^{(\phi)}, S_{1}^{(\phi)}$, and $S_{2}^{(\phi)}$ commute with the total number of photons $S_{0}^{(\phi)}$ (see (23) and (24)), the eigenstates and eigenvalues of these operators can be specified by the index $n={ } \mathrm{P}_{\mu} v_{\mu}$, describing the total number of photons in a given state (29), and by an additional index 1 , describing a given distribution of $n$ photons over three independent 'phase' components of the dipole radiation labelled by index $\mu$. The total number of possible different 1 , corresponding to a given $n$, is clearly

$$
\frac{1}{2}(n+2)(n+1) \text {. }
$$

All one can expect is that the eigenvalues of the operators (23) and (24) are represented as

$$
\begin{aligned}
\mathcal{E}^{(\phi)}\left|\phi_{l}^{(n)}\right\rangle & =\epsilon_{l}^{(n)} \mathrm{e}^{\mathrm{i} \varphi_{l}^{(n)}}\left|\phi_{l}^{(n)}\right\rangle \\
S_{0}^{(\phi)}\left|\phi_{l}^{(n)}\right\rangle & =n\left|\phi_{l}^{(n)}\right\rangle \\
S_{1}^{(\phi)}\left|\phi_{l}^{(n)}\right\rangle & =2 \epsilon_{l}^{(n)} \cos \varphi_{l}^{(n)}\left|\phi_{l}^{(n)}\right\rangle \\
S_{2}^{(\phi)}\left|\phi_{l}^{(n)}\right\rangle & =2 \epsilon_{l}^{(n)} \sin \varphi_{l}^{(n)}\left|\phi_{l}^{(n)}\right\rangle
\end{aligned}
$$

where $\mid \varphi_{1}{ }^{(n)} i$ is the eigenstate (29) at given $n$ and 1 . Note that this choice of the eigenvalues

(30) corresponds to the above interpretation of the Stokes operators $S_{1,2}^{(\phi)}$. In view of (24) and

(26) the modulus of the eigenvalues (30) can be determined as

$\left(\epsilon_{l}^{(n)}\right)^{2}=\left\langle\phi_{l}^{(n)}\left|\mathcal{E}^{(\phi)}\left(\mathcal{E}^{(\phi)}\right)^{+}\right| \phi_{l}^{(n)}\right\rangle=\sum_{\mu \mu^{\prime}} v_{\mu} v_{\mu^{\prime}} \mathrm{e}^{\mathrm{i}\left(\mu-\mu^{\prime}\right) 2 \pi / 3}$

$$
=\sum_{\mu} v_{\mu}^{2}-\left(v_{+} v_{0}+v_{0} v_{-}+v_{-} v_{+}\right)=n^{2}+2\left(v_{+}^{2}+v_{-}^{2}\right)-3 n\left(v_{+}+v_{-}\right)+3 v_{+} v_{-} .
$$

In turn, for the 'phase eigenvalues' $\phi$ we get

$$
\tan \varphi=\frac{\sqrt{3}\left(v_{+}-v_{-}\right)}{2 v_{0}-\left(v_{+}+v_{-}\right)}
$$

We now note that the cosine and sine of the radiation phase operators were defined in $[4,11]$ by the relations

$$
\mathrm{C}_{\mathrm{R}}=\mathrm{KS}_{1} \quad \mathrm{~S}_{\mathrm{R}}=\mathrm{KS}_{2}
$$

where the normalization coefficient $\mathrm{K}$ is determined from the condition 


$$
\mathrm{hCR} 2+\mathrm{SR} 2 \mathrm{i}=1
$$

where $h . . . \mathrm{i}$ is the averaging over an arbitrary state of the dipole radiation. One can see that condition (34) is similar to (31) in the case of averaging over the states $\left|\phi_{l}^{(n)}\right\rangle$. In this

\begin{tabular}{|c|c|c|c|c|}
\hline$n=1$ & $\epsilon^{(1)}=1$ & $\varphi_{l}^{(1)}=2 l \pi / 3$ & $l=0, \pm 1$ & \\
\hline$n=2$ & $\begin{array}{l}\epsilon^{(2)}=2 \\
\epsilon^{(2)}=1\end{array}$ & $\begin{aligned} \varphi_{l}^{(2)} & =2 l \pi / 3 \\
\varphi_{l}^{(2)} & =(2 l+1) \pi / 3\end{aligned}$ & $\begin{array}{l}l=0, \pm 1 \\
\quad l=0, \pm 1\end{array}$ & \\
\hline$n=3$ & $\begin{array}{l}\epsilon^{(3)}=3 \\
\epsilon^{(3)}=\sqrt{3}\end{array}$ & $\begin{aligned} \varphi_{l}^{(3)} & =2 l \pi / 3 \\
\varphi_{l}^{(3)} & =(2 l+1) \pi / 6\end{aligned}$ & $\begin{array}{l}l=0, \pm 1 \\
\quad l=0,1, \ldots\end{array}$ & $\ldots, 5$ \\
\hline$n=4$ & $\begin{array}{l}\epsilon^{(4)}=4 \\
\epsilon^{(4)}=\sqrt{7} \\
\epsilon^{(4)}=2 \\
\epsilon^{(4)}=1\end{array}$ & $\begin{array}{l}\varphi_{l}^{(4)}=2 l \pi / 3 \\
\varphi_{l}^{(4)}= \pm \tan ^{-1}(\sqrt{3} / \\
\varphi_{l}^{(4)}=(2 l+1) \pi / 3 \\
\varphi_{l}^{(4)}=2 l \pi / 3\end{array}$ & $\begin{array}{l}l=0, \pm 1 \\
5)+2 l \pi / 3 \\
\quad l=0, \pm 1 \\
l=0, \pm 1\end{array}$ & $l=0, \pm 1$ \\
\hline$n=5$ & $\begin{array}{l}\epsilon^{(5)}=5 \\
\epsilon^{(5)}=\sqrt{13} \\
\epsilon^{(5)}=\sqrt{7} \\
\epsilon^{(5)}=2 \\
\epsilon^{(5)}=1\end{array}$ & $\begin{array}{l}\varphi_{l}^{(5)}=2 l \pi / 3 \\
\varphi_{l}^{(5)}=\tan ^{-1}(\sqrt{3} / 7) \\
\varphi_{l}^{(5)}= \pm \tan ^{-1}(\sqrt{3} / \\
\varphi_{l}^{(5)}= \pm \tan ^{-1}(\sqrt{3} / \\
\varphi_{l}^{(5)}=2 l \pi / 3 \\
\varphi_{l}^{(5)}=(2 l+1) \pi / 3\end{array}$ & $\begin{array}{l}l=0, \pm 1 \\
\pm 2 l \pi / 3 \\
7)+2 l \pi / 3 \\
5)+\pi+2 l \pi / 3 \\
l=0, \pm 1 \\
\quad l=0, \pm 1\end{array}$ & $\begin{array}{l}l=0, \pm 1 \\
l=0, \pm 1 \\
\quad l=0, \pm 1\end{array}$ \\
\hline
\end{tabular}

case $K=\left(2 \epsilon_{l}^{(n)}\right)^{-1}$. Hence, the phase states $\left|\phi_{l}^{(n)}\right\rangle_{(29)}$ are the eigenstates of the radiation cosine and sine operators (33). It is interesting that the eigenvalues of the phase variable $\phi_{1}{ }^{(n)}$ determined by (32) belong to the interval $(0,2 \pi)$ and form a discrete set at any $n$ and $1=$ $1,2, \ldots,(n+1)(n+2) / 2$. The first few eigenvalues are shown in table 1 and figure 1 .

It is not difficult to see that the averaging of (33) with respect to the vacuum state gives

$$
\operatorname{hvac}\left|\mathrm{C}_{\mathrm{R}}\right| \text { vaci }=\text { hvac }\left|\mathrm{S}_{\mathrm{R}}\right| \text { vaci }=0
$$

while the variances are $V_{v a c}\left(C_{R}\right)=V_{v a c}\left(S_{R}\right)=\frac{1}{2}$. Hence, as one can expect, the vacuum distribution of the radiation phase is uniform. A similar result can be also obtained in the case of a single-mode coherent state $\mid \alpha_{\mu} \mathrm{i}$.

Consider, again, the coherent state $\mid \alpha_{+} ; 0 ; \alpha-i$ at $\left|\alpha_{+}\right|=\left|\alpha_{-}\right| \equiv|\alpha|$, which was discussed in sections 3 and 4 . In this case, condition (34) gives

$$
\mathrm{K}=\left[4|\alpha|^{2}\left(2+|\alpha|^{2}\right)\right]-^{1 / 2}
$$

so that 


$$
\begin{aligned}
\left\langle C_{R}\right\rangle & =\frac{|\alpha| \cos \Delta_{+-}}{\sqrt{2+|\alpha|^{2}}} \\
\left\langle S_{R}\right\rangle & =\frac{|\alpha| \sin \Delta_{+-}}{\sqrt{2+|\alpha|^{2}}}
\end{aligned}
$$

where $1_{+^{-}}$has the same meaning as above. One can see that at $|\alpha|^{2} \rightarrow \infty$ we get $h C_{R} i \rightarrow \cos 1_{+^{-}}$ and $\mathrm{hS}_{\mathrm{R}} \mathrm{i} \rightarrow \sin 1_{+^{-}}$. In this limit, the variances

$$
V\left(C_{R}\right)=V\left(S_{R}\right)=\frac{2+\cos \Delta_{+-}}{2\left(2+|\alpha|^{2}\right)}
$$

tend to zero. Hence, the radiation phase (33) has the natural classical limit.
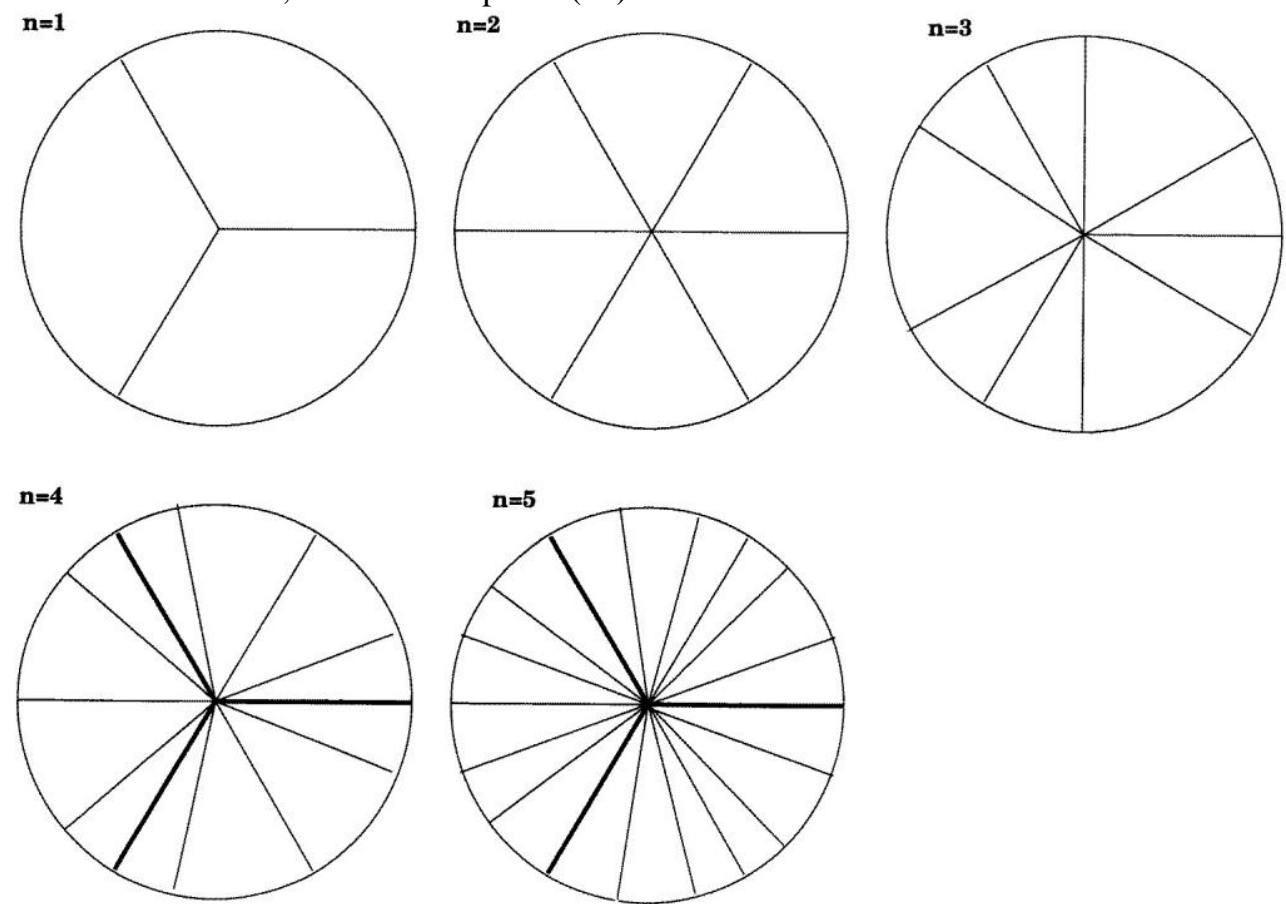

Figure 1. The structure of eigenvalues (32). The bold lines correspond to the double-degenerated eigenvalues.

Employing the formulae obtained at the end of section 4 then gives

$$
\left|\alpha_{+} ; 0 ; \alpha_{-}\right\rangle=\prod_{\mu=-1}^{1} \mathrm{e}^{-\left|\alpha_{\mu}^{(A)}\right|^{2} / 2} \sum_{v_{\mu}=0}^{\infty} \frac{\left(\alpha_{\mu}^{(A)}\right)^{v_{\mu}}}{\sqrt{v_{\mu} !}}\left|v_{\mu}\right\rangle \text {. }
$$

Taking into account that $\left.\mathrm{P}_{\mu}\right|_{\mu} ^{\alpha}{ }_{\mu}$ (A) $\left.\right|^{2}=\left.\left.{ }^{2}\right|^{\alpha}\right|^{2}$, for the probability that the radiation field can be observed in an arbitrary phase state (25) we get 
$\left|\left\langle v_{+} ; v_{0} ; v_{-} \mid \alpha_{+} ; 0 ; \alpha_{-}\right\rangle\right|^{2}=\mathrm{e}^{-2|\alpha|^{2}}\left(\frac{2}{3}\right)^{n} \frac{|\alpha|^{2 n}}{v_{+}^{!} v_{0} ! \nu_{-} !} \prod_{\mu=-1}^{1}\left[1+\cos \left(\Delta_{+-}-2 \mu \pi / 3\right)\right]^{v_{\mu}}$

where $^{2} n=P_{\mu} v_{\mu}$. One can see from (35) that this probability tends to zero when $|\alpha|^{2} \rightarrow 0$ or $|\alpha|$ $\rightarrow \infty$. This means that the eigenvalues of the radiation phase are distributed uniformly over the interval $(0,2 \pi)$ in the vacuum states as well as in the classical limit of high intensity $|\alpha|^{2}$. Between these two extrema, the probability (35) has a maximum which might be considerably high. It is interesting that the position of the maximum is completely determined by the mean number of photons $\left|\alpha_{\max }\right|^{2}=\mathrm{n}$, while the magnitude depends also on the phase difference $1_{+^{-}}$ (see figure 2).

We now note that the probability to have a given radiation phase in the coherent state under consideration is much higher. Consider, for example, the eigenvalue of the radiation phase $\phi=2 \pi / 3$. Employing equations (31) and (32) then gives the following properties of the states corresponding to this radiation phase:

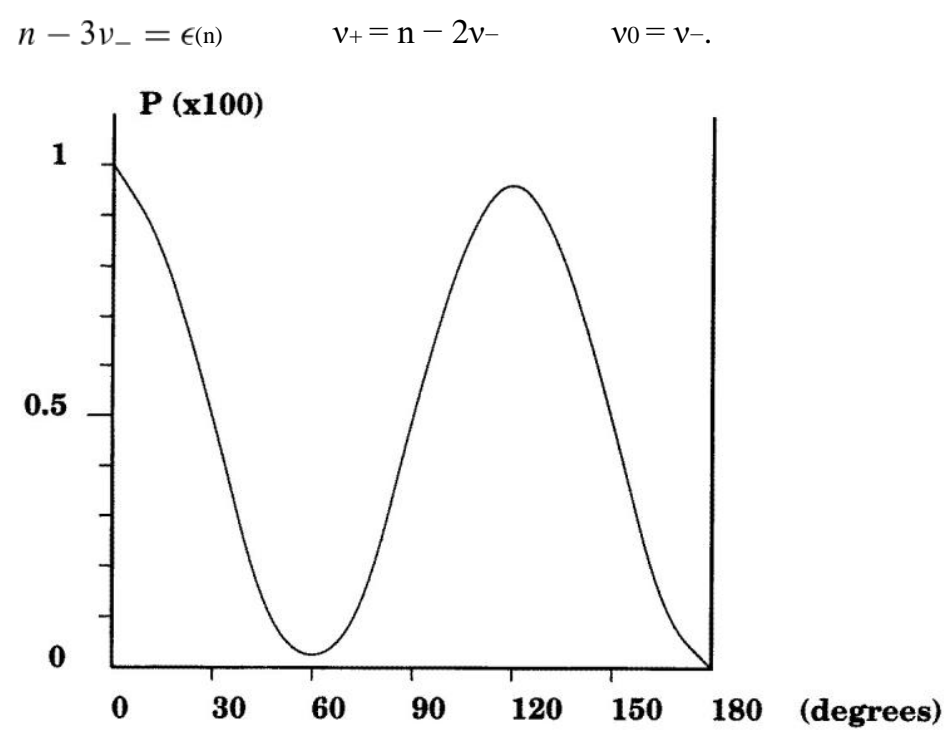

Figure 2. Probability (35) versus $1_{+^{-}}$at $v_{+}=v_{0}, v_{-}=1$.

It is easy to see that the states obeying these conditions have the following structure:

$$
\mathrm{n}_{\mathrm{p}}-2 \mathrm{k}_{\mathrm{p}} ; \mathrm{k}_{\mathrm{p}} ; \mathrm{k}_{\mathrm{p}} \mathrm{i}
$$

where $\mathrm{p}$ is an integer and for each $\mathrm{n}_{\mathrm{p}}=3 \mathrm{p}, 3 \mathrm{p}-1,3 \mathrm{p}-2$ the numbers $\mathrm{k}_{\mathrm{p}}$ take the values $\mathrm{k}_{\mathrm{p}}=$ $0,1, \ldots, p-1$. For example, at $n_{p}=10$ we get $p=4$ and $n_{p}=3 p-2$, while the states $(36)$ are

$$
|10 ; 0 ; 0 \mathrm{i} \quad| 8 ; 1 ; 1 \mathrm{i} \quad|6 ; 2 ; 2 \mathrm{i} \quad| 4 ; 3 ; 3 \mathrm{i} .
$$


Consider first the states $\mid \mathrm{n}_{\mathrm{p}} ; 0 ; 0 \mathrm{i}$ in (36). Then, the probability (35) takes the value

$\mathcal{P}_{n_{p}} \equiv\left|\left\langle n_{p} ; 0 ; 0 \mid \alpha_{+} ; 0 ; \alpha_{-}\right\rangle\right|^{2}=\mathrm{e}^{-2|\alpha|^{2}}\left(\frac{2}{3}\right)^{n_{p}} \frac{|\alpha|^{2 n_{p}}}{n_{p} !}\left[1+\cos \left(\Delta_{+-}-2 \pi / 3\right)\right]^{n_{p}}$.

It is clear that $\mathrm{P}_{\mathrm{p}}$ reaches its maximum at $1_{+^{-}}=2 \pi / 3$. Then the total probability of the states $\mid \mathrm{n}_{\mathrm{p}} ; 0 ; 0 \mathrm{i}$ is

$$
\mathcal{P}=\sum_{n_{p}=1}^{\infty} \mathcal{P}_{n_{p}}=\mathrm{e}^{-2|\alpha|^{2} / 3}-\mathrm{e}^{-2|\alpha|^{2}}
$$

(see figure 3). It is clear that $\mathrm{P}$ gives the lower bound of the total probability to have the

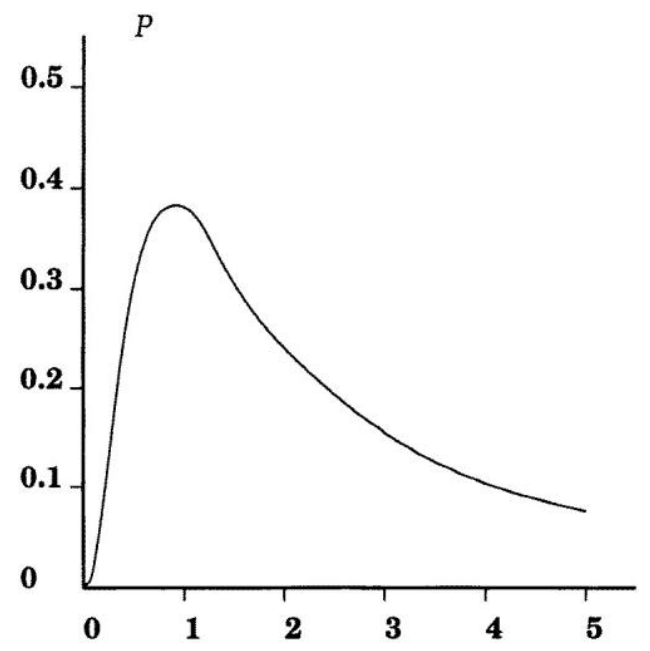

radiation phase $\phi=2 \pi / 3$ in the coherent state $\mid \alpha_{+} ; 0 ; \alpha-i$. The contribution of the other states

(36) can be calculated in the same way.

\section{Conclusion}

Let us briefly discuss our results. We have studied the angular momentum and polarization of the electric dipole radiation by an atom or molecule. We have shown that the quantum description of the polarization differs essentially from the classical picture. Although the latter is based on the five generalized Stokes parameters (15), the former needs the nine Stokes operators (16) and (19) which are represented by linear combinations of the generators (18) of the SU(3) sub-algebra in the Weyl-Heisenberg algebra (8) of the dipole photons and by the total number of photons. These nine Stokes operators describe the physical quantities with

Figure 3. Lower boundary of probability to have the radiation phase $\phi=2 \pi / 3$ as a function of $|\alpha|^{2}$ at $1+^{-}=2 \pi / 3$.

essentially different quantum fluctuations. Two of the Stokes operators describing the phase information in (16) and (19), precisely $S_{1}$ and $S_{2}$, form the representation of the Cartan algebra in SU(3) (18). Hence, the corresponding physical quantities can be measured at once. From the physical point of view these two operators, apart from a normalization factor, give the cosine and sine of the radiation phase (35).

The polar decomposition of the SU(2) algebra proposed by Vourdas [2] for a quantum mechanical system is not possible in the case of the angular momentum ME (9) of the dipole radiation. To determine the quantum phase states we have suggested a different approach, based on the canonical transformation (22) of the photon operators and further definition of the radiation phase states (25) as the number states of the dual photons. We have shown that the radiation phase states $(25)$ are the eigenstates of the cosine and sine of the radiation phase operators. The discrete eigenvalues of the phase variable, determined by (31)-(34), lie in the interval $(0,2 \pi)$ and cover it uniformly in the classical limit provided by the coherent state of the radiation field of high intensity. In the quantum domain of low intensities the probability 
to observe a given eigenvalue of the radiation phase is calculated. Let us stress that, unlike the Pegg-Barnet phase (or phase difference), the radiation phase is determined here by operators (35) in the whole Hilbert space directly. The measurement of the Stokes operators $\mathrm{S}_{1}, \mathrm{~S}_{2}$ or the radiation phase through the use of an eight-port homodyne detector has been briefly discussed in [11]. Detailed investigation of the detection of spherical photons as well as the use of another scheme (e.g., a six-port homodyne detector [17]) needs further discussion.

The most important result in the field of quantum phase was obtained by Mandel et al [10]. According to their analysis, there is no unique quantum phase variable, describing universally themeasuredphasepropertiesoflight. Thisverystrongstatementobtainedatotallyconvincing confirmation in a number of recent experiments $[10,16]$. In general, the quantum phase variables can be divided into two classes. First of all, we have the pure operational phases which are completely determined by the scheme of measurement. In addition, there might be some inherent quantum phases related to the quantum properties of photons and obtained in the process of generation. Since a photon can be specified by itsenergy, angular momentum and/or linear momentum, the inherent phase should be determined by either the angular momentum or linear momentum as the energy is a scalar. The former is connected with the spin state and hence, with the polarization. This inherent quantum phase is just the radiation phase (35). The latter can lead to some geometrical phase, which, for example, can be measured as the phase difference between two plane waves generated by one source in opposite directions.

We also note that both the operational phases and the radiation phase are determined in terms of bilinear Hermitian forms in the photon operators. At first sight, such a definition runs counter to the original idea by Dirac to determine the Hermitian quantum phase via linear forms in the photon operators [18] (for a recent review see [9, 19]). Leaving aside Dirac's problem of existence of a Hermitian quantum phase variable of a harmonic oscillator, we should emphasize that the use of bilinear forms seems to be quite reasonable from the physical point of view. It can be argued in the following way. First, the vacuum field has no phase at all. This is the same as saying that the vacuum state of photons is degenerated with respect to the phase or that the phase is distributed uniformly over the vacuum. Thus, the inherent quantum phase of a photon is generated by a source. This is not an unusual assumption. Actually, the classical amplitudes of a multipole field are completely determined by the source functions [5]. Hence, the multipole photon operators, which are obtained by the quantization of the classical amplitudes [1], are also specified by the source (e.g., see [20] and the discussion in [21]). This means that the properties of a photon depend on the quantum properties of the source. The 'information'aboutthesourceistransmittedtothephotonviatheconservationlaws(ofenergy, linear momentum and angular momentum) which are always expressed in terms of bilinear Hermitian forms in the photon operators. The 'phase information' should be transmitted in the same way. This statement can be illustrated using the Jaynes-Cummings model of section 2. Using the phase states (4) one can introduce the dual atomic operators

$$
\left.R_{\mu \mu^{\prime}}^{(\phi)} \equiv \| \phi_{\mu}\right\rangle\left\langle\phi_{\mu^{\prime}}\left\|\quad \mathrm{R}_{\mu \mathrm{G}(\varphi)} \equiv\right\| \varphi \mu \mathrm{ihG} \| .\right.
$$

Then, the simultaneous use of the dual representation of the atomic operators and the canonical transformation (22) leads to the following form of the Hamiltonian (10):

$$
H^{(\phi)}=\sum_{\mu=-1}^{1}\left[\omega A_{\mu}^{+} A_{\mu}+\omega_{0} R_{\mu \mu}^{(\phi)}+g\left(R_{\mu G}^{(\phi)} A_{\mu}+\text { H.c. }\right)\right.
$$


which has exactly the same operator structure as (10). Since the atomic operators $R^{(\varphi)}$ describe here the transitions between the atomic phase states, and the operators $\mathrm{A}, \mathrm{A}^{+}$determine the annihilation and creation of photons with given radiation phase, the interaction term in (37) describes the transmission of the quantum phase information from the atom to photons.

Inturn, thedetectionprocessisalsobasedonthetransmissionofenergy, linearmomentum or angular momentum from the photons to a detecting device. That is why the operational phasesarealsodeterminedintermsofthebilinearHermitianformsinthephotonoperators[10]. By virtue of the above discussion, one can unify the operational idea [10] with that of our approach [4] to determine the phase in terms of what can be transmitted from a quantum mechanical system to photons and vice versa.

Our consideration of radiation phase of the dipole radiation is based on the use of spherical photons determined as the quantum counterpart of rotational invariant solutions of the wave equation [1]. This means that the radiation is described in terms of photons with given energy and angular momentum. At the same time, in the usual formulation of quantum optics, the radiation field is considered in terms of states of photons with given energy and linear momentum, corresponding to the translational invariant solutions of the homogeneous wave equation (e.g., see $[15,21]$ ). Since the components of the angular and linear momenta do not commute, the two representations of the quantum electromagnetic field are different in principle. In particular, this difference is manifested in the presence of a radial (longitudinal) component of the quantum multipole field, while the plane waves of photons are always transversal with respect to the direction of the linear momentum. The a priori neglect of the radial component leads to violation of symmetry, i.e. to a change of the representation. In turn, the consistent consideration of this component leads to a more general picture of polarization based on the SU(3) rather than SU(2) algebra of Stokes operators. It is due to the existence of the radial component that the phase-dependent Stokes operators $S_{1}$ and $S_{2}$ in (16), as well as the cosine and sine of the radiation phase operators (33), commute with each and hence can be measured at once. We showed in section 3 that the presence of the radial component even in the vacuum state contributes into the quantum fluctuations of Stokes parameters. Let us note that a similar contribution affects some other physical quantities as well. As a particular example, the intensity of radiation with given polarization at given point $\mathrm{rE}$ can be mentioned [22]. In fact, the electric field (13) can be also represented as follows [13]:

$$
\begin{aligned}
\mathrm{E}(\mathrm{E} \mathrm{r}) \mathrm{E} & =\mathrm{X} \chi \mathrm{E}_{\mu} \mathrm{X} \mathrm{V}_{\mu \mathrm{m}}(\mathrm{r}) \mathrm{aE} \mathrm{m} \\
\mu=-1 \quad \mathrm{~m}=-1 &
\end{aligned}
$$

where $\vec{\chi}_{\mu}$ is the unit vector, corresponding to a spin state of photon. It is then clear that the local intensity

$$
\mathrm{I}_{\mu}(\mathrm{r}) \mathrm{E}=\mathrm{hE}_{\mu}^{+}(\mathrm{r}) \mathrm{EE} \mu(\mathrm{r}) \mathrm{E} \mathrm{i}
$$

contains the off-diagonal terms of the form $\left\langle a_{+}^{+} a_{0}\right\rangle,\left\langle a_{0}^{+} a_{-}\right\rangle$and conjugated terms. Hence, the variance of intensity should contain an additional contribution, coming from these offdiagonal terms.

Our considerations so far have been applied to the electric dipole radiation. As a final remark, we note that the canonical transformation (22) can be generalized to the case of arbitrary pure $(\mathrm{j}, \mathrm{m})$ multipole radiation as follows: 


$$
A_{j \mu}=\frac{1}{\sqrt{2 j+1}} \sum_{m=-1}^{1} \mathrm{e}^{\mathrm{i} m \phi_{j \mu}} a_{j m} \quad \phi_{j \mu} \equiv \frac{2 \mu \pi}{\sqrt{2 j+1}} .
$$

Usingtransformation(38)onecandeterminetheradiationphasestatesofanarbitrarymultipole radiation in the same way as $(25)$.

\section{References}

[1] Heitler W 1984 The Quantum Theory of Radiation (New York: Dover)

[2] Vourdas A 1990 Phys. Rev. A 411653

[3] Serre J-P 1977 Linear Representations of Finite Groups (New York: Springer)

[4] Shumovsky A S 1997 Opt. Commun. 136219

[5] Jackson J D 1975 Classical Electrodynamics (New York: Wiley)

[6] Shumovsky A S and Mustecaplıo“ glu־ O E 1997" Phys. Lett. A 235438

[7] Levi-Leblond J M 1979 Ann. Phys., NY 101319

Barnett S M and Pegg D T 1990 Phys. Rev. A 413427

Sanchez-Soto L L and Luis A 1994' Opt. Commun. 10584

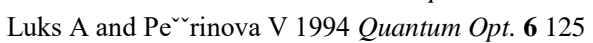

[8] Pegg D T and Barnett S M 1988 Europhys. Lett. 6483

Barnett S M and Pegg D T 1989 J. Mod. Opt. 367

[9] Pegg D T and Barnett S M 1997 J. Mod. Opt. 44225

[10] Noh J W, Fougeres A and Mandel L 1991 Phys. Rev. Lett. 671426

Noh J W, Fougeres A and Mandel L 1992`Phys. Rev. A 45242

[11] Shumovsky A S and Mustecaplı“" glu־ O E 1998" Opt. Commun. 146124 Shumovsky A S and Mustecaplı“" gluॅ O E 1998“ J. Mod. Opt. 45619

[12] Shumovsky A S and Mustecaplı“" glu־ O E 1998“" Phys. Rev. Lett. 801202

[13] Berestetskii V B, Lifshitz E M and Pitaevskii L D 1982 Quantum Electrodynamics (Oxford: Pergamon)

[14] Luis A and Sanchez-Soto L L 1993' Phys. Rev. A 484702

Luis A, Sanchez-Soto L L and Tana's R 1995'Phys. Rev. A 511634

[15] Mandel L and Wolf E 1995 Optical Coherence and Quantum Optics (Cambridge: Cambridge University Press) [16] Fougeres A, Torgerson J R and Mandel L 1994'

Opt. Commun. 105199

Torgerson J R and Mandel L 1996 Phys. Rev. Lett. 763939

Torgerson J R and Mandel L 1997 Opt. Commun. 133153

[17] Zucchetti A, Vogel W and Welsch D-G 1996 Phys. Rev. A 54856

[18] Dirac P A M 1927 Proc. R. Soc. A 114243

[19] Carruthers P and Nieto M M 1968 Rev. Mod. Phys. 40411

Lynch R 1995 Phys. Rep. 256367

[20] Khoo I C and Eberly J H 1976 Phys. Rev. A 142174

[21] Scully M O and Zubairy M S 1997 Quantum Optics (Cambridge: Cambridge University Press)

[22] Shumovsky A S and Klyachko A A 1999 Phys. Rev. Lett. submitted 